\title{
An MDP Based Energy Efficient Transmission Policy for Wireless Terminals
}

\author{
Xiao-Hui Lin, Yi-De Huang, Chao Yang, Ning Xie, Bin Chen, Shengli Zhang, Hui Wang \\ Faculty of Information Engineering, ShenZhen University, China \\ Email: xhlin@szu.edu.cn \\ Received July, 2013
}

\begin{abstract}
In wireless network, terminals are usually energy constrained. In order to extend the lifetime of the terminal, the limited energy must be utilized in an efficient manner. In this paper, under the constant transmission power scenario, we propose an Energy Efficient Transmission Policy (EETP) which is derived by using Markov Decision Process (MDP). The simulation results show that compared with the Threshold Transmission Policy (TTP), the proposed policy can reduce the energy consumption significantly, while satisfying the performance demand at the same time.
\end{abstract}

Keywords: Energy Efficient; Transmission Policy; MDP; Tradeoff

\section{Introduction}

Wireless communication systems have deeply penetrated into our daily life and the wireless terminals such as smart phone, tablet PC and wireless sensor node have brought fundamental changes to our society. However, the design of wireless system is a challenging task, in which, many issues must be properly handled. Among these design issues, one of the most crucial one is energy-efficiency of wireless system.

In literature, there have been a lot of research works on the design of energy-efficient communication systems. The design philosophy is usually to achieve the required level of performance by consuming just enough energy.Specifically, in [1], Uysal-Biyikoglu formulates the problem as how to minimize the energy consumption while satisfying the delay constraints at the same time. In order to reduce the energy consumption, In [2] based on the queue length in the system, Curt Schurgers proposed a traffic adaptive technique called Dynamic Modulation Scaling (DMS) which adaptively change the modulation level to lower the overall energy consumption, while bounding the packet delay at an acceptable level. $\operatorname{In}[3]$, Baris Ata studies how to control the transmitting power based on the buffer state. The objective is to minimize long-run average energy consumption subject to a QoS constraint, which is expressed as an upper bound on the packet loss rate. Author also formulates the issue into a Lagrangian problem and derives the optimal power control policy.

In [4], Liu analyzes the cross-layer design of AMC system. Specifically, both the queue length and channel state have been considered. By deriving the stationary probability of the system state, Liu gives a performance analysis model and develops a cross-layer design method. The work is novel, but the author doesn't consider the energy issue. In this paper, we adopt the Liu's method in the performance analysis and try to design an energy efficient transmission system.

\section{System Model}

\subsection{Markov Model for Wireless Channels and AMC}

In this paper, we focus on the uplink transmission. Terminals transmit data to base station through wireless channel. We assume the channel is a block fading channel. It means that the channel is frequency flat, and remains invariant per frame, but is allowed to vary from frame to frame, which is suitable for slowing varying wireless channel. And we adopt Finite State Markov Channel (FSMC) model to describe the channel. FSMC is a popular model adopted in literature [4-6]. Specifically, the channel quality can be captured by the received signal-to-noise ratio (SNR). By partitioning the range of the received SNR into a finite number of intervals, a finite-state model for the fading channel is built [7]. We use $\left\{S_{n}\right\}, n=0,1, \ldots N$ to denote the different state of the channel, and let $\mathrm{A}_{0}<\mathrm{A}_{1}<\mathrm{A}_{2} \cdots<\mathrm{A}_{\mathrm{N}+1}$ be the thresholds of different state. If the instantaneous received SNR is between $A_{k}$ and $A_{k+1}$, we will say the channel is in state $S_{k}$. Based on the method in [4-7], we can get the channel state transition probability matrix $\mathrm{P}_{\mathrm{c}}$. 
In our study, we adopt the adaptive physical layer design called ABICM [8], in which variable throughput modulator and channel coding are used. We assume a 4-mode AMC configuration is used. Therefore, there are four distinctive throughputs available as listed in Table 1. We divide the channel into five different states $\left\{\mathrm{S}_{0} ; \mathrm{S}_{1}\right.$; $\left.\mathrm{S}_{2} ; \mathrm{S}_{3} ; \mathrm{S}_{4}\right\}$ according to the instantaneous channel quality. Note that when channel is in state $S_{0}$, no packet is sent because the channel is in deep fading. So if the feedback CSI falls within the interval $\left\{\mathrm{A}_{\mathrm{k}} ; \mathrm{A}_{\mathrm{k}+1}\right\} \mathrm{k}=0,1,2,3,4$, transmission mode $\mathrm{k}$ is selected.

The processing unit at the data link layer is packet that includes multiple information bits. And each packet at this layer is assumed to contain a fixed number of bits (NP). At physical layer, the information delivery is performed in a frame-by-frame manner. And in physical layer, the symbol rate (RS) and the frame duration are assumed to be constant, which means that each frame contains a fixed number of symbols (Ns).

Therefore, based on the modulation and coding rate pair adopted in Table 1, we can find that the number of packets one frame can carry is $1: 2: 4: 8$. Since the transmit power is constant, the energy needed to transmit one packet under different modes is $8: 4: 2: 1$. So we should transmit in higher mode as much as possible to conserve energy.

\subsection{Buffer Queuing Analysis}

In [4], authors originally propose a discrete time queuing analysis method which is also used in the performance analysis in our paper, with some emendations made to fit the situations in our work. The queuing model is illustrated in Figure 1.

Table 1. AMC system.

\begin{tabular}{ccccc}
\hline & Mode1 & Mode2 & Mode3 & Mode4 \\
\hline Modulation & QPSKK & 8PSK & 32QAM & 512QAM \\
Coding rate & 1 & 2 & 4 & 8 \\
bits/symbol & 2 & 3 & 5 & 9 \\
ThresholdAk & 1 & 2 & 4 & 8 \\
\hline
\end{tabular}

Number or arrival paciets
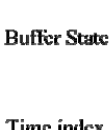

Bn-1

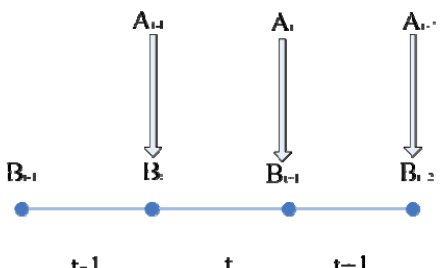

Channel Statc

C.:

C:

$C_{i-1}$

$\mathrm{G}_{\mathrm{l}-\mathrm{I}}$

G.

$\mathbf{G}_{1-1}$
Let $t$ denote the time units and $A_{t}$ the number of packets arriving at time $t$. The time unit in our study is frame duration $\mathrm{T}_{\mathrm{f}}$. For simplicity, let the arrival process be

$$
\mathrm{A}_{\mathrm{t}}=a \text { for all } t
$$

Let $B_{t}$ denote the buffer state which is the number of packets currently stored in the buffer. Thus we have

$$
B_{t} \in \mathbf{B} \quad \mathbf{B}=\{0,1,2,3 \cdots \mathrm{K}\}
$$

We use $C_{t}$ to denote the channel state at time $t$, which can be written as

$$
C_{t} \in \mathbf{C} \quad \mathbf{C}=\left\{\mathrm{S}_{0}, \mathrm{~S}_{1}, \mathrm{~S}_{2}, \cdots \mathrm{S}_{\mathrm{N}}\right\}
$$

In our study, we use buffer state $B_{t}$ and channel state $\mathrm{C}_{\mathrm{t}}$ to form a state pair $\left(\mathrm{C}_{t}, \mathrm{~B}_{\mathrm{t}}\right)$. The number of packet that one time unit can transmit is highly related with the system state $\left(C_{t}, B_{t}\right)$. We use $G_{t}$ to denote the action (i.e. number of packets to transmit) we can select at time $t$, thus we have

$$
G_{t} \in \mathbf{G} \quad \mathbf{G}=\left\{0,1, \cdots \min \left(\mathrm{C}_{\mathrm{t}}, \mathrm{B}_{\mathrm{t}}\right)\right\}
$$

At any time $t$, based on the $B_{t}, C_{t}, G_{t}$ and $A_{t}$, we can get $\mathrm{B}_{\mathrm{t}+1}$ by

$$
\mathrm{B}_{\mathrm{t}+1}=\mathrm{B}_{\mathrm{t}}-\mathrm{G}_{\mathrm{t}}+\mathrm{A}_{\mathrm{t}}
$$

Then we can get the queue state transition probability as

$$
\operatorname{Pb}\left(B_{t+1} \mid\left(C_{t}, B_{t}\right), G_{t}\right)=\left\{\begin{array}{l}
1 \text { if } G_{t} \in G \& B_{j}=B_{i}+A_{t}-G_{t} \\
0 \text { otherwise }
\end{array}\right.
$$

$\mathrm{G}_{\mathrm{t}}$ can be decided by the transmission policy $\mathrm{g}$, which indicates how many packets we should transmit under the current system state. When we have the system state $\left(\mathrm{C}_{\mathrm{t}}, \mathrm{B}_{\mathrm{t}}\right)$, guided by the transmission policy $\mathrm{g}$, we can get the transmission decision $\mathrm{G}_{\mathrm{t}}=\mathrm{g}\left(\mathrm{C}_{\mathrm{t}}, \mathrm{B}_{\mathrm{t}}\right)$. Then the buffer transition probability (6) can be calculated.

In order to understand the system better, we need to derive the system state transition probability and stationary distribution. Since the channel process and queue process are independent with each other, based on $\mathrm{Pc}$ and (6) we can get the system state transition probability

$$
\begin{aligned}
& P\left(\left(C_{t}+1, B_{t}+1\right) \mid\left(C_{t}, B_{t}\right), G_{t}\right) \\
= & P_{C}\left(C_{t}+1 \mid C_{t}\right) \quad P_{B}\left(B_{t+1} \mid B_{t}, g\left(C_{t}, B_{t}\right)\right)
\end{aligned}
$$

Based on the method proposed in [4] (24-26), we can derive the stationary distribution $\operatorname{Pg}(\mathrm{c}, \mathrm{b}),(\mathrm{c}, \mathrm{b}) \in \mathrm{C} \times$ $\mathrm{B}$ under the transmission policy $\mathrm{g}$.

Then we can calculate the desired performance under different transmission policy and find the optimal one. The packet loss rate $\mathrm{Pd}$ is [4] (27-30):

$$
\begin{gathered}
E[D]=\sum \max [0, a-K+(b-g(c, b))] * P \mathbf{g}(c, b) \\
c \in C ; b \in B \\
P_{d}=\frac{E[D]}{a} P_{d}
\end{gathered}
$$

Figure 1. Queuing model. 
And the expected energy consumed per frame under the transmission policy $\mathbf{g}$ can be calculated by

$$
\tilde{E}=\sum_{(c, b) \in C \times B} P(c, b) E_{c} g(c, b)
$$

where Ec is the energy cost for transmit one packet under the channel state $\mathrm{c}$.

\section{MDP Formulation}

Based on the analysis given in previous sections, the system state can be described as a state pair $(\mathrm{Ct} ; \mathrm{Bt})$ where $\mathrm{Ct}$ is the channel state and $\mathrm{Bt}$ is the buffer state at time t. After we get the system state, we can select an action from the action space $\mathrm{Gt} \in \mathrm{G}=\{0 ; 1 ; \ldots$; $\left.\min \left(\mathrm{C}_{\mathrm{t}} ; \mathrm{B}_{\mathrm{t}}\right)\right\}$. And we know that the state transition probability depends on the action selected. Therefore, the problem can be modeled as a Markov Decision Process (MDP) [10]. Next we should construct a cost function to derive the optimal transmission policy by using MDP.

Since in the transmission policy design, we need to consider two factors-energy consumption and the level of QoS achieved, the cost function is constructed with both factors taken into consideration:

$$
R((c, b), g(c, b))=E_{c} * g(c, b)+\beta^{*} \frac{5^{2}}{K^{2}} *(b-g(c, b))^{2}
$$

Note that equation (11) is dimensionless. The first term in (11) is the energy cost for packet transmission with the transmission policy g. Since the buffer is finite, we assume that when the buffer is full, the newly arriving packets are simply dropped, which can incur packet loss and delay. Therefore the second term in (11) is a penalty cost. We replace the constraint on QoS with a penalty cost for packet storing. From the second term, we can see that the penalty cost is in quadratic growth with the number of packet stored. $\beta \in[0,1]$ is a weight factor, representing the relative importance of the QoS, therefore, the larger $\beta$ the higher QoS demand.

Our objective is to find a transmission policy $g *$ which can minimize the overall cost, which is called value function V. Specifically, optimal transmission policy can be written as:

$$
\begin{aligned}
\mathbf{g}^{*} & =\arg \min _{\mathbf{g} \in \Pi} \mathbf{V}^{\mathbf{g}} \\
& =\arg \min _{\mathbf{g} \in \Pi} E_{(c, b)}^{\mathbf{g}}\left[\sum_{t=0}^{\infty} \lambda^{t} R\left(\left(c_{t}, b_{t}\right), g\left(c_{t}, b_{t}\right)\right)\right]
\end{aligned}
$$

where $\Pi$ is all possible policy space, and $\lambda$ is a discount factor. Since the system evolves in a stochastic manner, we should calculate the expectation of overall cost. We let $\operatorname{Eg}(\mathrm{c}, \mathrm{b})$ denotes the expectation cost under the policy g. The policy g* above is called the optimal policy, and the related cost function $\mathrm{Vg} *$ under the policy $g *$ is called the optimal discounted value function.
This problem is an infinite horizon discounted cost problem. It can be solved by the numerical method called Policy Iteration Algorithm.

\section{Numerical Simulation Results}

In this section, we perform extensive simulations to validate the proposed policy. The simulation parameters are listed in Table 2:

The weight factor $\beta$ in (11) indicates the QoS demand. We vary the value of $\beta$ from 0 to 1 . Based on the method we propose in section 3, we get the optimal transmission policies with different $\beta$. Then the system transition probability (7) and stationary distribution of the system can be calculated. With the stationary distribution, we can evaluate the performance of the optimal transmission policy under different $\beta$ by using (9-10). Then we get the packet loss rate and expected energy consumed under the different QoS demand $\beta$. The impact of different under different average received SNR is illustrated in Figures 2-5.

Table 2. Simulation parameters for.

\begin{tabular}{ll}
\hline Parameter & Value \\
\hline Symbol Rate $\mathrm{R}_{\mathrm{s}}$ & $200 \mathrm{ksymbol} / \mathrm{s}$ \\
Packet Size $\mathrm{L}_{\mathrm{p}}$ & $4000 \mathrm{bit}$ \\
Frame Duration $\mathrm{T}_{\mathrm{f}}$ & $20 \mathrm{~ms}$ \\
Dopple Frequency $\mathrm{f}_{\mathrm{d}}$ & $2 \mathrm{~Hz}$ \\
Buffer Size K & 5 \\
Arrival Process Parameter a & 1 \\
Average received SNR $\Upsilon_{0}$ & $\Upsilon_{0} \in[10,19]$ \\
Initial Battery Energy & 100 Joule \\
ES $_{4}$ & $10^{-3}$ Joule
\end{tabular}

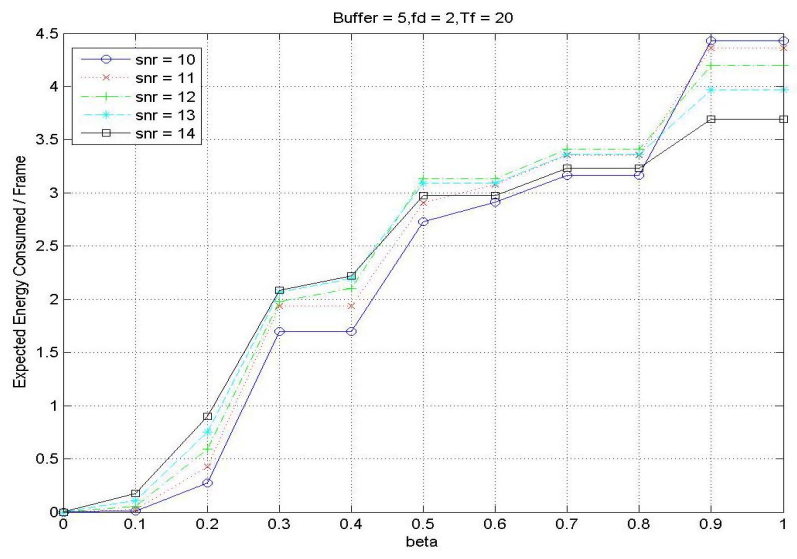

Figure 2. Expected energy consumed per frame versus beta (Average received SNR=[10,11,12,13,14]). 


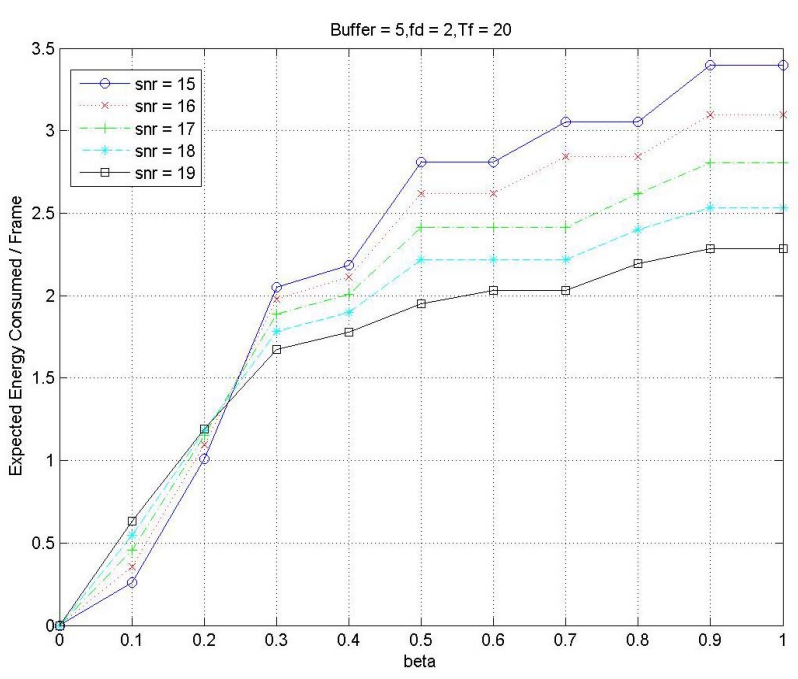

Figure 3. Expected energy consumed per frame versus beta (Average received SNR $=[15,16,17,18,19])$.

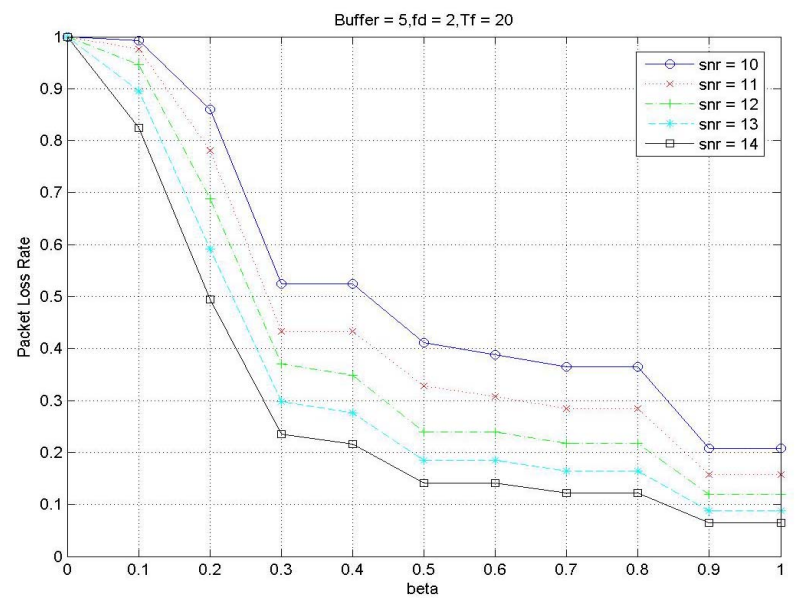

Figure 4. Packet loss rate versus beta(Average received SNR $=[10,11,12,13,14])$.

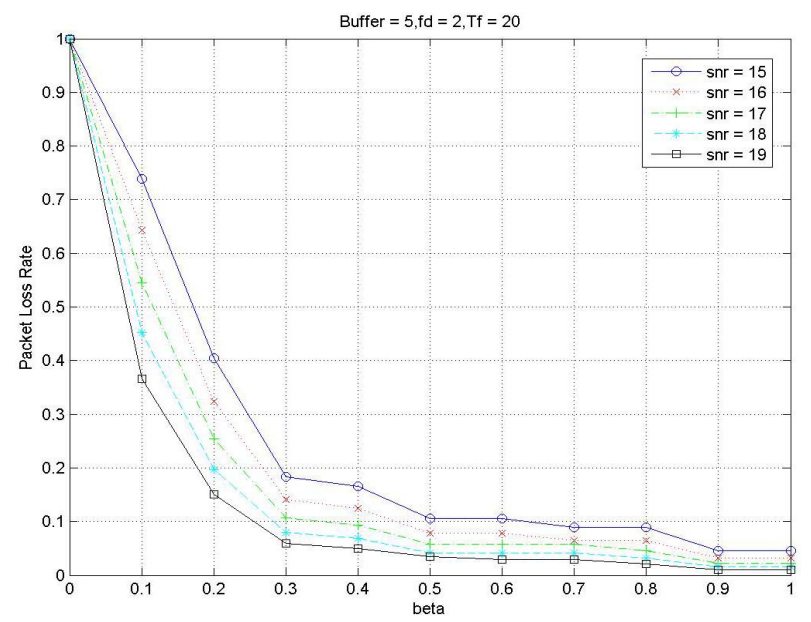

Figure 5. Packet loss rate versus beta (Average received SNR $=[15,16,17,18,19])$.
In the Figure2-3 we can observe that the expected energy consumed per frame increases with $\beta$, which indicates that when the QoS demand increases, the penalty for buffering the packet increases as well. It is unwise to store the packet in buffer, because the storing cost is higher than that of transmission cost, even we transmit the packet under a low mode. Hence system should transmit packet as much as possible no matter what transmit mode it would adopt. However when become small, the storing cost is relatively smaller than the transmit cost. Therefore, system would prefer packet storing rather than transmission, and wait until the channel quality recover.

From the Figures 4-5 we can observe that the packet loss rate decreases with $\beta$, which means that by varying $\beta$, we can achieve different levels of QoS. Joint with Figures 2-3 we can see the tradeoff between energy and QoS, i.e. Higher energy consumption means a better QoS.

Next, we compare our Energy-Efficient Transmission Policy (EETP) with Threshold Transmission Policy (TTP) [8].

The average received SNR $=15 \mathrm{~dB}$. Under the Threshold Transmission Policy, only when the received SNR is above the threshold $A_{k}$ can we transmit packets. The system transmits packet in a best effort manner. Then we have 4 different transmission policies under the Threshold Transmission Policy, among which, we can select the best one.

However, compared with TTP, more policy strategies are provided in our method, and thus search space is much larger. Consequently, we can further reduce the energy consumption with better policy as illustrated in Figure 6.

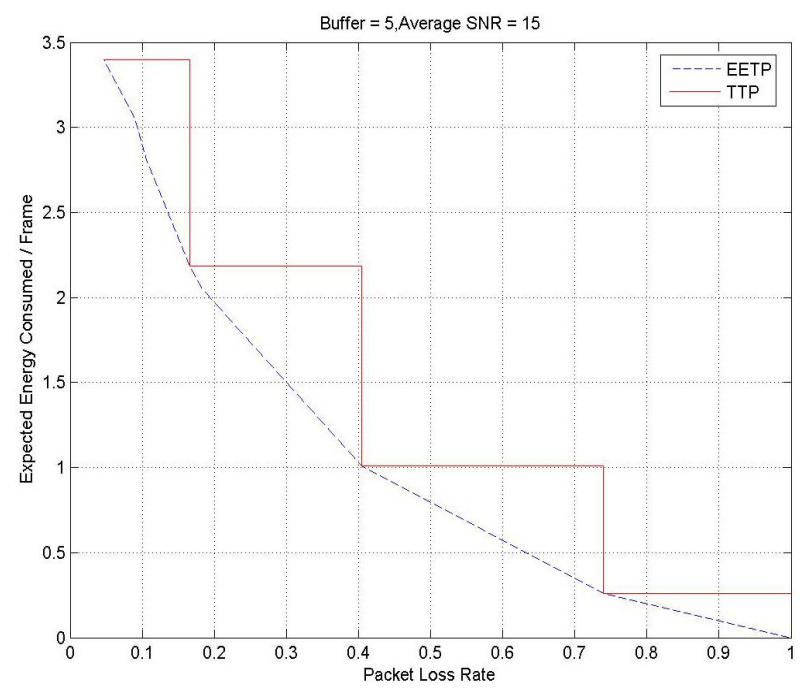

Figure 6. Energy Efficient Transmission Policy versus Threshold Transmission Policy. 


\section{Conclusions}

In this paper, we study the energy efficient transmission under the constant transmission power constraint. We formulate the Energy Efficient Transmission Problem as a MDP problem and use the Policy Iteration Algorithm to get the optimal transmission policy which consumes the least amount of energy while achieving the QoS demand. We compare our method with previous threshold method. Simulation results show that our transmission policy can reduce the energy consumption significantly.

\section{Acknowledgements}

The research was jointly supported by research grant from Natural Science Foundation of China under project number 61171071, 60602066, 60902016, 61001182 and 60773203, 973 Program under the project number 2013CB336700, and grants from Foundation of Shenzhen City under project number JC200903120069A, JC201005280556A, JC201005250035A, JC201005250047A， JCYJ20120613115037732， and ZDSY20120612094614154. The Corresponding author of the paper.

\section{REFERENCES}

[1] E. Uysal-Biyikoglu, B. Prabhakar and A. El Gamal, 'Energy Efficient Packet Transmission over a Wireless Link," IEEE/ACM Transactions on Networking, Vol. 10, 2002, pp. 487-499. doi:10.1109/TNET.2002.801419

[2] S. Curt, R. Vijay and B. S. Mani, "Power Management for Energy-aware Communication Sincerely yours, ys- tems." Vol. 2, 2003, pp. 431-447.

[3] B. Ata, "Dynamic Power Control in a Wireless Static Channel Subject to a Quality-of-Service Constraint," $O p$ erations Research, Vol. 53, 2005, pp. 842-851. doi:10.1287/opre.1040.0188

[4] L. Qingwen, Z. Shengli and G. B. Giannakis, "Queuing with Adaptive Modulation and Coding over Wireless Links: Cross-Layer Analysis and Design," IEEE Transactions on Wireless Communications, Vol. 4, 2005, pp. 1142-1153. doi:10.1109/TWC.2005.847005

[5] J. Razavilar, K. J. R. Liu, and S. I. Marcus, "Jointly optimized bit-rate/delay control policy for wireless packet networks with fading channels," IEEE Transactions on Communications, Vol. 50, 2002, pp. 484-494. doi:10.1109/26.990910

[6] H. Xinwei and K. Shoraby, "A Dynamic Programming Approach for Optimal Scheduling Policy in Wireless Networks," In Proceeding of. Eleventh International Conference on Computer Communications and Networks, 2002, pp. 530-536.

[7] W. H. Shen and N. Moayeri, "Finite-state Markov Channel Model for Radio Communication Channels," IEEE Transactions on Vehicular Technology, Vol. 44, 1995, pp. 163-171.doi:10.1109/25.350282

[8] X. -H. Lin, K. Yu-Kwong and W. Hui, "Cross-layer Design for Energy Efficient Communication in Wireless Sensor Networks," Vol. 9: John Wiley and Sons Ltd., 2009, pp. 251-268.

[9] C. S. Taek and A. J. Goldsmith, "Degrees of Freedom in Adaptive Modulation: A Unified View," IEEE Transactions on Communications, Vol. 49, 2001, pp. 1561-1571. doi: $10.1109 / 26.950343$

[10] M. L. Puterman, Dynamic Programming, 3rd ed. Vol 4, 2002. 